\title{
FRANKL-RÖDL-TYPE THEOREMS FOR CODES AND PERMUTATIONS
}

\author{
PETER KEEVASH AND EOIN LONG
}

\begin{abstract}
We give a new proof of the Frankl-Rödl theorem on forbidden intersections, via the probabilistic method of dependent random choice. Our method extends to codes with forbidden distances, where over large alphabets our bound is significantly better than that obtained by Frankl and Rödl. We also apply our bound to a question of Ellis on sets of permutations with forbidden distances and to establish a weak form of a conjecture of Alon, Shpilka and Umans on sunflowers.
\end{abstract}

\section{INTRODUCTION}

A family $\mathcal{A}$ of sets is said to be $l$-avoiding if $|A \cap B| \neq l$ for all $A, B \in \mathcal{A}$. Erdös conjectured (see 9]) that for any $\epsilon \in(0,1)$ there is $\delta=\delta(\epsilon)>0$ such that given $l$ with $\epsilon n \leq l \leq(1 / 2-\epsilon) n$, any $l$-avoiding family $\mathcal{A} \subset \mathcal{P}[n]$ satisfies $|\mathcal{A}| \leq(2-\delta)^{n}$ and offered $\$ 250$ for a solution. In [14, Frankl and Rödl gave a positive answer to Erdös' conjecture, proving a stronger result for $k$-uniform $l$-avoiding families. Note that for $A, B \in\left(\begin{array}{c}{[n]} \\ k\end{array}\right)$, their intersection satisfies $\max (0,2 k-n) \leq|A \cap B| \leq k$. Now it is easily seen that if $l \geq k-o(n)$ or $l \leq \max (0,2 k-n)+o(n)$, then there exist $l$-avoiding families $\mathcal{A} \subset\left(\begin{array}{c}{[n]} \\ k\end{array}\right)$ with $|\mathcal{A}|=(1-o(1))^{n}\left(\begin{array}{l}n \\ k\end{array}\right)$. Frankl and Rödl showed that for $l$ in between these extremes, all $k$-uniform $l$-avoiding families have exponentially small density.

Theorem 1.1 (Frankl-Rödl). Let $\alpha, \epsilon \in(0,1)$ with $\epsilon \leq \alpha / 2$. Let $k=\lfloor\alpha n\rfloor$ and $l \in[\max (0,2 k-n)+\epsilon n, k-\epsilon n]$. Then any l-avoiding family $\mathcal{A} \subset\left(\begin{array}{c}{[n]} \\ k\end{array}\right)$ satisfies $|\mathcal{A}| \leq(1-\delta)^{n}\left(\begin{array}{l}n \\ k\end{array}\right)$ where $\delta=\delta(\alpha, \epsilon)>0$.

Theorem 1.1 along with several extensions of the theorem proved in 14 have had a huge impact in a number of different areas, including discrete geometry [15], communication complexity [20] and quantum computing [6].

In Section 2 of this paper we give a new proof of Theorem 1.1. We show that the theorem can in fact be deduced from an earlier theorem due to Frankl and Wilson (see Theorem 2.3 below). While our new proof of Theorem 1.1 does not seem to improve on the bounds given in [14, the same proof method does significantly improve bounds when we forbid distances over a larger underlying alphabet. Given $q \in \mathbb{N}, q \geq 2$, we will say that a set $\mathcal{C}$ is a $q$-ary code if $\mathcal{C} \subset[q]^{n}$. The Hamming distance between two words $x, y \in[q]^{n}$ is written as $d_{H}(x, y)=\left|\left\{i \in[n]: x_{i} \neq y_{i}\right\}\right|$. For a code $\mathcal{C}$ we write $d(\mathcal{C})=\left\{d_{H}(x, y)\right.$ : distinct $\left.x, y \in \mathcal{C}\right\} \subset[n]$. Frankl and Rödl used Theorem 1.1 to prove the following result.

Received by the editors February 25, 2014 and, in revised form, February 12, 2015.

2010 Mathematics Subject Classification. Primary 05D05; Secondary 05D40, 94B65.

This research was supported in part by ERC grant 239696 and EPSRC grant EP/G056730/1. 
Theorem 1.2 (Frankl-Rödl). Let $\mathcal{C} \subset[q]^{n}$, and let $\epsilon$ satisfy $0<\epsilon<1 / 2$. Suppose that $\epsilon n<d<(1-\epsilon) n$, and $d$ is even if $q=2$. If $d \notin d(\mathcal{C})$, then $|\mathcal{C}| \leq(q-\delta)^{n}$ with some positive constant $\delta=\delta(\epsilon, q)$.

(Note that in order for Theorem 1.2 to hold for $q=2$, we must have that $d$ is even since the set $\mathcal{C}_{0}=\left\{x \in\{0,1\}^{n}: \sum_{i} x_{i} \equiv 0(\bmod 2)\right\}$ satisfies $\left|\mathcal{C}_{0}\right|=2^{n-1}$ but contains no odd distances.)

In Section 3, we improve this to the following:

Theorem 1.3. Let $\mathcal{C} \subset[q]^{n}$, and let $\epsilon$ satisfy $0<\epsilon<1 / 2$. Suppose that $\epsilon n<d<$ $(1-\epsilon) n$, and $d$ is even if $q=2$. If $d \notin d(\mathcal{C})$, then $|\mathcal{C}| \leq q^{(1-\delta) n}$ with some positive constant $\delta=\delta(\epsilon)$.

As a consequence of Theorem 1.3 we obtain a Frankl-Rödl-type theorem for permutations. Given two permutations $\pi, \rho \in S_{n}$ we write

$$
d_{S_{n}}(\pi, \rho)=|\{i \in[n]: \pi(i) \neq \rho(i)\}| \text {. }
$$

For a set $\mathcal{S} \subset S_{n}$ we write

$$
d_{S_{n}}(\mathcal{S})=\{d \in[n]: d(\pi, \rho)=d \text { for distinct } \pi, \rho \in \mathcal{S}\} .
$$

Recently Ellis [10] asked how large a family $\mathcal{S} \subset S_{n}$ can be if $d \notin d_{S_{n}}(\mathcal{S})$ for some $d \in[n]$. A result of Deza and Frankl [8] answers this question for $d=n$, showing that the largest such families have size $(n-1)$ !. Ellis [10] gave a tight upper bound of $(n-2)$ ! when $d=n-1$, provided $n$ is sufficiently large. Here we consider this question when $\epsilon n<d<(1-\epsilon) n$ for $\epsilon>0$. It is easily seen that for such $d$ there exist sets of permutations $\mathcal{S} \subset S_{n}$ with $d \notin d_{S_{n}}(\mathcal{S})$ such that $|\mathcal{S}| \geq(n !)^{c}$ where $c=c(\epsilon) \in(0,1)$. By taking $q=n$ and viewing permutations $\pi \in S_{n}$ as vectors in $[q]^{n}$, with $\pi=(\pi(1), \ldots, \pi(n))$, since $\left|S_{n}\right|=n !=q^{(1-o(1)) n}$, Theorem 1.3 has the following consequence:

Theorem 1.4. Let $\mathcal{S} \subset S_{n}$, and let $\epsilon$ satisfy $0<\epsilon<1 / 2$. Suppose that $\epsilon n<d<$ $(1-\epsilon) n$. If $d \notin d_{S_{n}}(\mathcal{S})$, then $|\mathcal{S}|<(n !)^{(1-\delta)}$ with some positive constant $\delta=\delta(\epsilon)$.

Before we discuss another consequence of Theorem 1.3, we need the following definition.

Definition 1.5. Given $v, w \in[q]^{n}$ let $\operatorname{Agree}(v, w)=\left\{i \in[n]:(v)_{i}=(w)_{i}\right\}$. A collection of vectors $v_{1}, \ldots, v_{k} \in[q]^{n}$ is said to form a strong sunflower with $k$ petals in $[q]^{n}$ if there is a fixed set $S \subset[n]$ such that $\operatorname{Agree}\left(v_{i}, v_{j}\right)=S$ for all distinct $i, j \in[k]$. A collection of vectors $v_{1}, \ldots, v_{k} \in[q]^{n}$ is said to form a weak sunflower with $k$ petals in $[q]^{n}$ if there is $D \in \mathbb{N}$ such that $\left|\operatorname{Agree}\left(v_{i}, v_{j}\right)\right|=D$ for all distinct $i, j \in[k]$.

Using Theorem 1.1, Frankl and Rödl proved that for any $k \in \mathbb{N}$ there exists $\delta=\delta(k)>0$ such that if $\mathcal{A} \subset\{0,1\}^{n}$ with $|\mathcal{A}|>(2-\delta)^{n}$, then $\mathcal{A}$ contains a weak sunflower with $k$ petals. Similarly, using the methods from 14 it can be shown that for any $k \in \mathbb{N}$ there exists $\delta=\delta(q, k)>0$ so that given a code $\mathcal{C} \subset[q]^{n}$ with $|\mathcal{C}| \geq(q-\delta)^{n}, \mathcal{C}$ contains a weak $k$-petal sunflower in $[q]^{n}$. In Section 4 we prove the following:

Theorem 1.6. Given $k \in \mathbb{N}$, there exists $\delta=\delta(k)>0$ such that the following holds. For $q \geq 2$, every $\mathcal{C} \subset[q]^{n}$ which does not contain a weak sunflower with $k$ petals satisfies $|\mathcal{C}| \leq q^{(1-\delta) n}$. 
This might be seen as giving evidence to a recent conjecture of Alon, Shpilka and Umans 2, who asked for a similar bound on families not containing a strong sunflower with 3 petals in $[q]^{n}$.

A crucial idea in the original proof of Theorem 1.1, along with an ingenious density increment argument, was to prove a stronger result. In [14 the authors actually proved a cross-intersecting version of Theorem 1.1.

Theorem 1.7 (Frankl-Rödl). Let $\alpha, \epsilon \in(0,1)$ with $\epsilon \leq \alpha / 2$. Let $k=\lfloor\alpha n\rfloor$ and $l \in[\max (0,2 k-n)+\epsilon n, k-\epsilon n]$. Then if $\mathcal{A}_{1}, \mathcal{A}_{2} \subset\left(\begin{array}{c}{[n]} \\ k\end{array}\right)$ with $\left|A_{1} \cap A_{2}\right| \neq l$ for all $A_{i} \in \mathcal{A}_{i}$, they satisfy $\left|\mathcal{A}_{1}\right|\left|\mathcal{A}_{2}\right| \leq(1-\delta)^{n}\left(\begin{array}{l}n \\ k\end{array}\right)^{2}$, where $\delta=\delta(\alpha, \epsilon)>0$.

We draw attention to the fact that the corresponding cross-versions of Theorem 1.3 and Theorem 1.4 with our improved bounds do not hold in general. Indeed, for even $n$, if we take $\mathcal{A}_{1}$ to be the collection of all permutations in $S_{n}$ sending $[n / 2]$ to $[n / 2]$ and $\mathcal{A}_{2}$ to be the collection of all permutations in $S_{n}$ sending $[n / 2]$ to $[n / 2+1, n]$, we see that $\left|\mathcal{A}_{i}\right| \geq(n / 2) !^{2} \geq n ! / 3^{n}=(n !)^{1-o(1)}$ but $d_{S_{n}}\left(\rho_{1}, \rho_{2}\right)=n$ for all $\rho_{i} \in \mathcal{A}_{i}$.

However, in Section 5 we give a simple condition which guarantees fixed distances between such sets.

Theorem 1.8. Given $\epsilon \in(0,1 / 2)$ there exists $\delta^{\prime}, \gamma>0$ such that the following holds. Let $q \geq 3$ and suppose that $\mathcal{C}, \mathcal{D} \subset[q]^{n}$ with $|\mathcal{C}| \geq q^{\left(1-\delta^{\prime}\right) n}$ and such that for all $x \in \mathcal{C}$ there exists $y \in \mathcal{D}$ with $d_{H}(x, y) \leq \gamma n$. Then given any $d \in(\epsilon n,(1-\epsilon) n)$, there exists $x \in \mathcal{C}$ and $y \in \mathcal{D}$ with $d_{H}(x, y)=d$.

Using Theorem 1.8 in combination with some isoperimetric results, we recover a version of Frankl and Rödl's cross-distance result for fixed $q$.

Corollary 1.9. Given $\epsilon \in(0,1 / 2)$ and $q \geq 3$ there exists $\delta=\delta(\epsilon, q)>0$ such that the following holds. Suppose that $\mathcal{C}, \mathcal{D} \subset[q]^{n}$ with $|\mathcal{C}||\mathcal{D}|>(q-\delta)^{2 n}$. Then given any $d \in(\epsilon n,(1-\epsilon) n)$, there exist $x \in \mathcal{C}$ and $y \in \mathcal{D}$ with $d_{H}(x, y)=d$.

Lastly, note that given $d \in[n]$ and any $x \in[q]^{n}$, there are exactly $\left(\begin{array}{l}n \\ d\end{array}\right)(q-1)^{d}$ words $y \in[q]^{n}$ with $d_{H}(x, y)=d$. In Section 6 , we prove a supersaturated version of Theorem 1.3 (which is essentially best possible):

Theorem 1.10. Given $\epsilon, \eta \in(0,1 / 2)$ there is $\delta^{\prime}>0$ such that the following holds. Let $\mathcal{C} \subset[q]^{n}$ with $|\mathcal{C}|>q^{\left(1-\delta^{\prime}\right) n}$ and $d \in \mathbb{N}$ with $\epsilon n<d<(1-\epsilon) n$ (and $d$ even if $q=2)$. Then there are at least $\left(\begin{array}{l}n \\ d\end{array}\right)(q-1)^{d}|\mathcal{C}| q^{-\eta n}$ pairs $x, y \in \mathcal{C}$ with $d_{H}(x, y)=d$.

Notation. Given a set $X, \mathcal{P}(X)$ will denote the power set of $X$ and $\left(\begin{array}{l}X \\ k\end{array}\right)$ will denote the collection of all subsets of size $k$ in $X$. Given $m, n \in \mathbb{N}$ with $m \leq n,[n]=$ $\{1, \ldots, n\}$ and $[m, n]=\{m, \ldots, n\}$. We also write $(n)_{m}$ for the falling factorial $(n)_{m}=n(n-1) \cdots(n-m+1)$.

\section{ForbidDING ONE INTERSECTION}

In this section we give our new proof of Theorem 1.1. Before beginning, we outline the argument for the proof. In Lemma 2.2 below, we will show that given upper bounds on the size of $k_{i}$-uniform and $l_{i}$-avoiding families on $n_{i}$ vertices for $i=1,2$, it is possible to obtain upper bounds on the size of $\left(k_{1}+k_{2}\right)$-uniform and $\left(l_{1}+l_{2}\right)$-avoiding families on $\left(n_{1}+n_{2}\right)$ vertices. The second tool we will need in the proof is the Frankl-Wilson theorem (see Theorem 2.3 and Corollary 2.4 below), 
which shows that the conclusion of Theorem 1.1 holds true for $k$-uniform, $l$-avoiding families whenever $k-l$ is a prime.

Suppose now that $k, l$ and $n$ are given as in the statement of Theorem 1.1, and assume that $k-l$ is odd. Let us choose $k_{1}, k_{2}, k_{3} \in \mathbb{N}$ and $n_{1}, n_{2}, n_{3} \in \mathbb{N}$ with $\left|k_{i}-k / 3\right|<1$ and $\left|n_{i}-n / 3\right|<1$ so that $k=\sum_{i=1}^{3} k_{i}$ and $n=\sum_{i=1}^{3} n_{i}$. We will also use a Vinogradov-type result due to Baker and Harman (see Theorem 2.5 below) which shows that any odd integer $m$ can be written as a sum of three primes $m=a_{1}+a_{2}+a_{3}$, where $\left|a_{i}-m / 3\right|=o(m)$. Applying this to $k-l$ we find three primes $k-l=a_{1}+a_{2}+a_{3}$ with $\left|a_{i}-(k-l) / 3\right|=o(k-l)=o(n)$. Now set $l_{i}=k_{i}-a_{i}$ for all $i \in[3]$. Since $k_{i}-l_{i}=a_{i}$ is prime for all $i$, by the Frankl-Wilson theorem we know that Theorem 1.1 holds for $k_{i}$-uniform and $l_{i}$-avoiding families on $n_{i}$ vertices, for all $i \in[3]$. Using Lemma 2.2 this will show that Theorem 1.1 holds for $\left(k_{1}+k_{2}\right)$ uniform and $\left(l_{1}+l_{2}\right)$-avoiding families on $\left(n_{1}+n_{2}\right)$ vertices. By applying Lemma 2.2 once again, we obtain that Theorem 1.1 holds for $k$-uniform and $l$-avoiding families on $n$ vertices, since $k=\sum_{i=1}^{3} k_{i}, n=\sum_{i=1}^{3} n_{i}$ and $l=\sum_{i=1}^{3} l_{i}$. The case when $k-l$ is even follows similarly, by writing $k-l$ as a sum of four primes.

To prove Lemma 2.2 we will make use of the probabilistic technique known as dependent random choice. The reader is directed to the recent survey of Fox and Sudakov [11, where many other interesting applications of the method are discussed. The following lemma gives a statement of the method which we will use in our applications. We include the short proof for convenience.

Lemma 2.1. Suppose that $G=(X, Y, E)$ is a bipartite graph with $|X|=M,|Y|=$ $N$ and $|E|=\alpha M N$. Then, for any $t \in \mathbb{N}$, there exists $X^{\prime} \subset X$ with $\left|X^{\prime}\right| \geq \alpha^{t} M / 2$ with the property that for all $x_{1}, x_{2} \in X^{\prime}$ we have $\left|N_{G}\left(x_{1}\right) \cap N_{G}\left(x_{2}\right)\right| \geq \alpha M^{-1 / t} N$.

Proof. To begin, choose uniformly at random $t$ elements $T$ with replacement from $Y$ and let $S$ denote the set of elements adjacent to all elements of $T$. By linearity of expectation

$$
\mathbb{E}(|S|)=\sum_{x \in X}\left(\frac{\left|N_{G}(x)\right|}{|Y|}\right)^{t} \geq \alpha^{t} M
$$

where the inequality follows from the convexity of the function $f(z)=z^{t}$.

We will say that a pair $x, x^{\prime}$ in $S$ is bad if $\left|N_{G}(x) \cap N_{G}\left(x^{\prime}\right)\right|<\alpha M^{-1 / t} N$. Now any bad pair has probability at most $\left(\frac{\left|N_{G}\left(x_{1}\right) \cap N_{G}\left(x_{2}\right)\right|}{N}\right)^{t} \leq \alpha^{t} M^{-1}$ of appearing in $S$. Therefore, letting $Z$ denote the number of bad pairs in $S$, we find that

$$
\mathbb{E}(Z) \leq \alpha^{t} M^{-1}\left(\begin{array}{c}
|X| \\
2
\end{array}\right) \leq \alpha^{t} M / 2
$$

In particular, $\mathbb{E}(|S|-Z) \geq \alpha^{t} M / 2$. Fix a choice of $T$ such that $|S|-Z$ is at least this big and delete one element from each bad pair $x_{1}, x_{2}$ in $S$. Taking $X^{\prime}$ to be the remaining set, we have $\left|X^{\prime}\right| \geq|S|-Z \geq \alpha^{t} M / 2$ and no pairs in $X^{\prime}$ are bad, as required.

The next lemma shows how one can use Lemma 2.1 to build fixed intersections from smaller ones.

Lemma 2.2. For $i=1,2$, suppose that $n_{i}, k_{i}, l_{i} \in \mathbb{N}$ and $p_{i} \in(0,1)$ are such that any $l_{i}$-avoiding family $\mathcal{A}_{i} \subset\left(\begin{array}{c}{\left[n_{i}\right]} \\ k_{i}\end{array}\right)$ satisfies $\left|\mathcal{A}_{i}\right| \leq p_{i}\left(\begin{array}{c}n_{i} \\ k_{i}\end{array}\right)$. Suppose that $t \in \mathbb{N}$

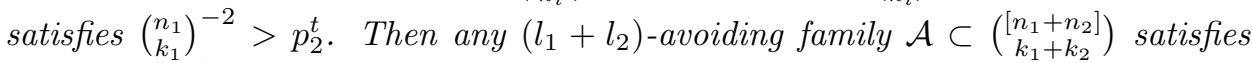
$|\mathcal{A}| \leq\left(2 p_{1}\right)^{1 / t}\left(\begin{array}{c}n_{1}+n_{2} \\ k_{1}+k_{2}\end{array}\right)$. 
Proof. Let $\mathcal{A} \subset\left(\begin{array}{c}{\left[n_{1}+n_{1}\right]} \\ k_{1}+k_{2}\end{array}\right)$ have size $|\mathcal{A}|=\alpha\left(\begin{array}{c}n_{1}+n_{2} \\ k_{1}+k_{2}\end{array}\right)$. We show that if $\alpha>\left(2 p_{1}\right)^{1 / t}$, then there exist $A, A^{\prime} \in \mathcal{A}$ with $\left|A \cap A^{\prime}\right|=l_{1}+l_{2}$.

To begin, choose a partition of $\left[n_{1}+n_{2}\right]$ uniformly at random into two sets $V_{1}$ and $V_{2}$ of size $n_{1}$ and $n_{2}$ respectively. Let $\mathcal{A}^{\prime} \subseteq \mathcal{A}$ denote the set

$$
\mathcal{A}^{\prime}=\left\{A \in \mathcal{A}:\left|A \cap V_{1}\right|=k_{1},\left|A \cap V_{2}\right|=k_{2}\right\}
$$

and let $Z$ denote the random variable $Z=\left|\mathcal{A}^{\prime}\right|$. It is easy to see that $\mathbb{E}(Z)=$ $\alpha\left(\begin{array}{l}n_{1} \\ k_{1}\end{array}\right)\left(\begin{array}{l}n_{2} \\ k_{2}\end{array}\right)$. We fix a partition $V_{1} \cup V_{2}=\left[n_{1}+n_{2}\right]$ for which $Z$ is at least this large.

Now we can view $\mathcal{A}^{\prime}$ as the edge set of a bipartite graph $G=(X, Y, E)$ with vertex bipartition $X=\left(\begin{array}{l}V_{1} \\ k_{1}\end{array}\right)$ and $Y=\left(\begin{array}{l}V_{2} \\ k_{2}\end{array}\right)$ in which $A B \in E(G)$ when $A \cup B \in \mathcal{A}^{\prime}$. We see that $G$ has at least $\alpha|X||Y|$ edges. Apply Lemma 2.1 to $G$ with $t$ as in the statement to find a set $X^{\prime} \subset X$ with $\left|X^{\prime}\right| \geq \alpha^{t}|X| / 2$ such that all distinct pairs $A_{1}, A_{2} \in X^{\prime}$ have at least $\alpha|X|^{-1 / t}|Y|$ common neighbours in $G$. Now if $\alpha>\left(2 p_{1}\right)^{1 / t}$, then $\left|X^{\prime}\right|>p_{1}\left(\begin{array}{l}n_{1} \\ k_{1}\end{array}\right)$ and by definition of $p_{1}$, we find $A_{1}, A_{2} \in X^{\prime}$ with $\left|A_{1} \cap A_{2}\right|=l_{1}$.

Let $\mathcal{B}^{\prime}$ denote the set of common neighbours of $A_{1}$ and $A_{2}$ in $G$. By Lemma 2.1 we find that

$$
\left|\mathcal{B}^{\prime}\right| \geq \alpha|X|^{-1 / t}|Y|>\left(2 p_{1}\right)^{1 / t}|X|^{-1 / t}|Y| \geq\left(\begin{array}{c}
n_{1} \\
k_{1}
\end{array}\right)^{-2 / t}|Y|>p_{2}\left(\begin{array}{c}
n_{2} \\
k_{2}
\end{array}\right) .
$$

The third inequality here holds since by definition of $p_{1}$ we have $p_{1} \geq 1 /\left(\begin{array}{l}n_{1} \\ k_{1}\end{array}\right)$, and the fourth holds as $\left(\begin{array}{l}n_{1} \\ k_{1}\end{array}\right)^{-2}>p_{2}^{t}$. But now by definition of $p_{2}$, there exists $B_{1}, B_{2} \in \mathcal{B}^{\prime}$ with $\left|B_{1} \cap B_{2}\right|=l_{2}$. By construction it can be seen that we have $A_{1} \cup B_{1}, A_{2} \cup B_{2} \in \mathcal{A}$ and clearly $\left|\left(A_{1} \cup B_{1}\right) \cap\left(A_{2} \cup B_{2}\right)\right|=l_{1}+l_{2}$, as required.

We will also make use of a theorem of Frankl and Wilson from [16.

Theorem 2.3 (Frankl-Wilson). Let $k, l \in \mathbb{N}$ such that $k-l$ is a prime power and $2 l+1 \leq k$. Suppose that $\mathcal{A} \subseteq\left(\begin{array}{c}{[n]} \\ k\end{array}\right)$ is an l-avoiding family. Then $|\mathcal{A}| \leq\left(\begin{array}{c}n \\ k-l-1\end{array}\right)$.

The following simple corollary of Theorem 2.3 will give us a slightly more convenient bound.

Corollary 2.4. Let $\epsilon \in(0,1)$ and let $l, k \in \mathbb{N}$ with $l<k$, such that $k-l$ is prime with $\max (0,2 k-n)+\epsilon n<l<k-\epsilon n$. Then any l-avoiding family $\mathcal{A} \subset\left(\begin{array}{c}{[n]} \\ k\end{array}\right)$ satisfies $|\mathcal{A}| \leq c^{n}\left(\begin{array}{l}n \\ k\end{array}\right)$ where $c=c(\epsilon)<1$.

Proof. Let $\mathcal{A}$ be an $l$-avoiding family with $|\mathcal{A}|=\alpha\left(\begin{array}{l}n \\ k\end{array}\right)$. By averaging, there exists a set $T \in\left(\begin{array}{c}{[n]} \\ l-\epsilon n\end{array}\right)$ such that $\mathcal{A}_{T}=\left\{A \in\left(\begin{array}{c}{[n] \backslash T} \\ k-|T|\end{array}\right): A \cup T \in \mathcal{A}\right\}$ has size $\left|\mathcal{A}_{T}\right| \geq \alpha\left(\begin{array}{c}n-|T| \\ k-|T|\end{array}\right)$. Setting $l^{\prime}=\epsilon n$ and $k^{\prime}=k-|T|$ it is easy to see that $\mathcal{A}_{T}$ is an $l^{\prime}$-avoiding $k^{\prime}$-uniform family. Since $k^{\prime}=k-l+\epsilon n \geq 2 \epsilon n+1=2 l^{\prime}+1$ and $k^{\prime}-l^{\prime}=k-l$ is prime, by Theorem 2.3, we have $\left|\mathcal{A}_{T}\right| \leq\left(\begin{array}{c}n-|T| \\ k^{\prime}-l^{\prime}\end{array}\right)=\left(\begin{array}{c}n-|T| \\ k-l\end{array}\right)$. This gives that

$$
\begin{aligned}
\alpha & \leq \frac{\left(\begin{array}{c}
n-|T| \\
k-l
\end{array}\right)}{\left(\begin{array}{l}
n-|T| \\
k-|T|
\end{array}\right)}=\frac{(k-|T|) !(n-k) !}{(k-l) !(n-k+\epsilon n) !} \\
& =\frac{(k-l+\epsilon n)_{\epsilon n}}{(n-k+\epsilon n)_{\epsilon n}} \leq\left(\frac{k-l+\epsilon n}{n-k+\epsilon n}\right)^{\epsilon n} \leq\left(\frac{1}{1+\epsilon}\right)^{\epsilon n}
\end{aligned}
$$

since $n-k \geq k-l+\epsilon n$. Taking $c=\left(\frac{1}{1+\epsilon}\right)^{\epsilon}<1$, the result follows. 
Lastly, we will use the following Vinogradov-type result due to Baker and Harman [5] which says that every large enough odd number can be written as a sum of three primes of almost equal size.

Theorem 2.5 (Baker-Harman). Every odd integer $n>n_{0}$ can be written as a sum of three primes $n=a_{1}+a_{2}+a_{3}$ with $\left|a_{i}-n / 3\right| \leq n^{4 / 7}$ for all $i$.

Proof of Theorem 1.1. Let $\mathcal{A} \subset\left(\begin{array}{c}{[n]} \\ k\end{array}\right)$ be an $l$-avoiding family which satisfies $l \in$ $[\max (0,2 k-n)+\epsilon n, k-\epsilon n]$. We wish to show that $|\mathcal{A}| \leq(1-\delta)^{n}\left(\begin{array}{l}n \\ k\end{array}\right)$, where $\delta=\delta(\alpha, \epsilon)>0$. By taking $\delta$ to be sufficiently small, we may assume that the theorem holds for small values of $n \leq n_{0}=n_{0}(\epsilon)$, so we will assume that $n \geq n_{0}$.

First suppose that $k-l$ is odd. Choose $k_{1}, k_{2}, k_{3} \in \mathbb{N}$ and $n_{1}, n_{2}, n_{3} \in \mathbb{N}$ with $\sum_{i=1}^{3} k_{i}=k$ and $\sum_{i=1}^{3} n_{i}=n$ with $\left|k_{i}-k / 3\right|<1$ and $\left|n_{i}-n / 3\right|<1$ for all $i$, with $n_{1} \geq n_{2} \geq n_{3}$. By Theorem 2.5, as $k-l>\epsilon n$ and $n>n_{0}(\epsilon)$, we can write $k-l=a_{1}+a_{2}+a_{3}$ where $a_{i}$ is prime and $\left|(k-l) / 3-a_{i}\right| \leq \frac{\epsilon n}{8}$ for all $i$. Also set $l_{i}=k_{i}-a_{i}$ for all $i$. Then $k_{i}-l_{i}$ is prime for all $i, \sum_{i} k_{i}-l_{i}=k-l$ and $\max \left(0,2 k_{i}-n_{i}\right)+\epsilon n_{i} / 2 \leq l_{i} \leq k_{i}-\epsilon n_{i} / 2$.

By Corollary 2.4 any $l_{i}$-avoiding family $\mathcal{A}_{i} \subset\left(\begin{array}{c}{\left[n_{i}\right]} \\ k_{i}\end{array}\right)$ satisfies $\left|\mathcal{A}_{i}\right| \leq p_{i}\left(\begin{array}{c}n_{i} \\ k_{i}\end{array}\right)$ where $p_{i}=c_{1}^{n_{i}}$ with $c_{1}=c(\epsilon / 2)<1$. Taking $t_{1}=\left\lceil 2 / \log _{2}\left(1 / c_{1}\right)\right\rceil$ we find that

$$
p_{2}^{t_{1}}=c_{1}^{t_{1} n_{2}} \leq 2^{-2 n_{1}}<\left(\begin{array}{l}
n_{1} \\
k_{1}
\end{array}\right)^{-2} .
$$

Therefore, by Lemma 2.2 any $\left(l_{1}+l_{2}\right)$-avoiding family $\mathcal{B} \subset\left(\begin{array}{c}{\left[n_{1}+n_{2}\right]} \\ k_{1}+k_{2}\end{array}\right)$ with $|\mathcal{B}|=$ $\beta\left(\begin{array}{c}n_{1}+n_{2} \\ k_{1}+k_{2}\end{array}\right)$ satisfies $\beta \leq\left(2 c_{1}^{n_{1}}\right)^{1 / t_{1}}$.

To complete the proof we simply repeat the previous argument. Let $t_{2}=$ $\left\lceil 4 t_{1} / \log _{2}\left(1 / c_{1}\right)\right\rceil$. Then we have

$$
\beta^{t_{2}} \leq\left(\left(2 c_{1}^{n_{1}}\right)^{1 / t_{1}}\right)^{t_{2}} \leq\left(2 c_{1}^{n_{1}}\right)^{4 / \log _{2}\left(1 / c_{1}\right)} \leq 2^{-2 n_{3}}<\left(\begin{array}{c}
n_{3} \\
k_{3}
\end{array}\right)^{-2},
$$

where the third inequality holds since $n \geq n_{0}(\epsilon)$. Lemma 2.2 now gives that any $l$-avoiding family $\mathcal{A} \subset\left(\begin{array}{c}{[n]} \\ k\end{array}\right)$ satisfies $|\mathcal{A}| \leq c_{2}^{n}\left(\begin{array}{c}n \\ k\end{array}\right)$ where $c_{2}=\left(c_{1}^{n_{3}}\right)^{1 / t_{2} n} \leq c_{1}^{1 / 4 t_{2}}<1$. As $c_{1}$ and $t_{2}$ depend only on $\epsilon$, this completes the proof in the case when $k-l$ is odd.

The case where $k-l$ is even can be proved by splitting $k-l$ into 4 primes of almost equal size. The proof now proceeds identically to the odd case, using an additional application of Lemma 2.2.

\section{Forbidding CODE Distances}

In this section we prove Theorem 1.3 We will assume that $q \geq 3$ throughout the section, as the case $q=2$ follows from Theorem 1.1

Given a set $V \subset[n]$, a vector $x \in[q]^{V}$ will be indexed by elements of $V$, i.e. $x=\left(x_{i}\right)_{i \in V}$. Given two disjoint sets $V$ and $W$ and vectors $x \in[q]^{V}$ and $y \in[q]^{W}$, we write $x \circ y$ for the vector $x \circ y \in[q]^{V \cup W}$, the concatenation of $x$ and $y$, given coordinate-wise by

$$
(x \circ y)_{i}= \begin{cases}x_{i} & \text { if } i \in V \\ y_{i} & \text { if } i \in W\end{cases}
$$


Given a collection of disjoint sets $V_{1}, \ldots, V_{k}$ and vectors $x_{i} \in[q]^{V_{i}}$ for all $i \in[k]$, we will write $x_{1} \circ x_{2} \cdots \circ x_{k} \in[q] \bigcup_{i} V_{i}$ to be the vector

$$
x_{1} \circ x_{2} \cdots \circ x_{k}=\left(\left(\left(x_{1} \circ x_{2}\right) \circ x_{3}\right) \circ \cdots \circ x_{k}\right) \text {. }
$$

(Note that this notation does not depend on the order in which the $x_{i}$ are taken.)

We also require the following definition:

Definition 3.1. Given a prime $p$ and a set $\mathcal{D} \subset \mathbb{Z}_{p} \backslash\{0\}$, we say that a code $\mathcal{C} \subset[q]^{n}$ is a $\mathcal{D}(\bmod p)$-code if for all $d \in d(\mathcal{C})$, we have $d \equiv d^{\prime} \bmod p$ for some $d^{\prime} \in \mathcal{D}$.

The following theorem, due to Frankl [12] (see also 4]), gives an upper bound on the size of $(\bmod p)$-codes.

Theorem 3.2 (Frankl). Suppose that $p$ is a prime and that $\mathcal{C} \subset[q]^{n}$ is a $\mathcal{D}$ $(\bmod p)$-code with $|\mathcal{D}|=l$. Then $|\mathcal{C}| \leq \sum_{i=0}^{l}\left(\begin{array}{c}n \\ i\end{array}\right)(q-1)^{i}$.

In applying Theorem 3.2 we use the following estimate due to Chernoff [7. Let $q \in \mathbb{N}$ with $q \geq 3$. Then given $\alpha \in(0,(q-1) / q)$, we have

$$
S_{q}(\alpha, n):=\sum_{i=0}^{\alpha n}\left(\begin{array}{c}
n \\
i
\end{array}\right)(q-1)^{i} \leq q^{f_{q}(\alpha) n}
$$

where $f_{q}(\alpha)=\alpha \log _{q}\left(\frac{q-1}{\alpha}\right)+(1-\alpha) \log _{q}\left(\frac{1}{1-\alpha}\right)$.

Proposition 3.3. For $q \geq 3$ and $\alpha \in[0,3 / 5]$ we have $S_{q}(\alpha, n) \leq q^{(1-1 / 125) n}$.

Proof. First note the following:

(1) $\frac{\partial f_{q}}{\partial \alpha}(\alpha)=\log _{q}\left[\frac{(q-1)(1-\alpha)}{\alpha}\right] \geq 0$ for $\alpha \in[0,(q-1) / q]$;

(2) $\frac{\partial^{2} f_{q}}{\partial \alpha^{2}}(\alpha)=\frac{1}{\log _{e} q}\left[-\frac{1}{1-\alpha}-\frac{1}{\alpha}\right] \leq 0$, so $f_{q}(\alpha)$ is concave as a function of $\alpha$ on $[0,1]$. As $f_{q}(0)=0$ and $f_{q}\left(\frac{q-1}{q}\right)=1$, this shows that $f_{q}(\alpha) \geq \frac{q \alpha}{q-1}$ for $\alpha \in[0,(q-1) / q]$

(3) $\frac{\partial f_{q}}{\partial q}(\alpha)=\frac{1}{q \log _{e} q}\left[\frac{q \alpha}{q-1}-f_{q}(\alpha)\right] \leq 0$ for $\alpha \in[0,(q-1) / q]$ by (2).

But then, for $q \geq 3$ and $\alpha \in[0,3 / 5] \subset[0,(q-1) / q]$, we have

$$
f_{q}(\alpha) \leq f_{3}(\alpha) \leq f_{3}(3 / 5) \leq 0.992,
$$

where the first inequality holds since $f_{q}(\alpha)$ is decreasing in $q$ by (3), the second since $f_{3}(\alpha)$ is increasing in $\alpha$ by (11) and the third by a numerical calculation.

Combined with Proposition 3.3 , Theorem 3.2 now gives the following corollary.

Corollary 3.4. Let $\epsilon \in(0,1)$ and $q \geq 3$. Suppose that $p$ is a prime with $\epsilon n<$ $p<3 n / 5$ and that $\mathcal{C} \subset[q]^{n}$ is a code with $p \notin d(\mathcal{C})$. Then $|\mathcal{C}| \leq q^{\left(1-\delta_{1}\right) n}$ where $\delta_{1}=\delta_{1}(\epsilon)>0$.

Proof. Suppose that $|\mathcal{C}|=\alpha q^{n}$. Choose $t$ so that $p \in\left(\frac{n-t}{2}, \frac{3(n-t)}{5}\right)$; this is possible by the stated bound on $p$ above. Now given a set $T \in\left(\begin{array}{c}{[n]} \\ t\end{array}\right)$ and elements $a_{i} \in[q]$ for $i \in T$, let

$$
\mathcal{C}_{T}=\left\{x \in \mathcal{C}: x_{i}=a_{i} \text { for all } i \in T\right\} .
$$

By averaging we find $T \in\left(\begin{array}{c}{[n]} \\ t\end{array}\right)$ and $\left\{a_{i} \in[q]: i \in T\right\}$ such that $\left|\mathcal{C}_{T}\right| \geq \alpha q^{n-t}$. View $\mathcal{C}_{T}$ as a subset of $[q]^{[n] \backslash T}$. Clearly $p \notin d\left(\mathcal{C}_{T}\right)$. Since $p>(n-t) / 2$, the set $\mathcal{C}_{T}$ is a 
$\mathcal{D}(\bmod p)$ code in $[q]^{[n] \backslash T}$, where $\mathcal{D}=\{1, \ldots, p-1\}$. Therefore by Theorem 3.2 and Proposition 3.3

$$
\alpha q^{n-t} \leq\left|\mathcal{C}_{T}\right| \leq S_{q}(3 / 5, n-t) \leq q^{(1-1 / 125)(n-t)} .
$$

Therefore $\alpha \leq q^{-(n-t) / 125} \leq q^{-\epsilon n / 125}$ using $\epsilon n \leq p \leq n-t$. Taking $\delta_{1}(\epsilon)=\epsilon / 125$ completes the proof.

Corollary 3.4 will allow us to deal with forbidden distances which are not too large. For larger distances we will use the following diametric theorem for $[q]^{n}$ due to Ahlswede and Khachatrian [1]. The diameter of a set $\mathcal{C} \subset[q]^{n}$, $\operatorname{diam}(\mathcal{C})$, is defined as

$$
\operatorname{diam}(\mathcal{C}):=\max \{d: d \in d(\mathcal{C})\}
$$

Given $t \in \mathbb{N}$ and $r \in \mathbb{N} \cup\{0\}$, let $\mathcal{K}_{r}(t) \subset[q]^{n}$ denote the set

$$
\mathcal{K}_{r}(t)=\left\{v \in[q]^{n}:\left|\left\{i \in[t+2 r]: v_{i}=1\right\}\right| \geq t+r\right\} .
$$

It is easy to see that $\operatorname{diam}\left(\mathcal{K}_{r}(t)\right)=n-t$ for all $r$. The following remarkable result shows that given $q$ and $t$, for some $r, \mathcal{K}_{r}(t)$ is the largest code in $[q]^{n}$ with diameter $n-t$.

Theorem 3.5 (Ahlswede, Khachatrian). Let $q, t \in \mathbb{N}$ with $q \geq 2$ and let $r \in \mathbb{N} \cup\{0\}$ be the largest integer such that

$$
t+2 r<\min \left\{n+1, t+2 \frac{t-1}{q-2}\right\} .
$$

Then any code $\mathcal{C} \subset[q]^{n}$ with $\operatorname{diam}(\mathcal{C}) \leq n-t$ satisfies $|\mathcal{C}| \leq\left|\mathcal{K}_{r}(t)\right|$. (By convention, $(t-1) /(q-2)=\infty$ if $q=2$.)

We will use the following simple consequence of Theorem 3.5

Corollary 3.6. Given $\epsilon \in(0,1 / 3)$ and $q \in \mathbb{N}$ with $q \geq 3$, every set $\mathcal{C} \subset[q]^{n}$ with $\operatorname{diam}(\mathcal{C}) \leq(1-\epsilon) n$ satisfies $|\mathcal{C}| \leq q^{\left(1-\delta_{2}\right) n}$ where $\delta_{2}=\delta_{2}(\epsilon)>0$.

Proof. Let $t=\epsilon n$. Since $\epsilon<1 / 3$, we have

$$
n+1>\epsilon n+2 \epsilon n-1 \geq t+2 \frac{t-1}{q-2},
$$

so the minimum in (3.1) is attained by the right hand term and gives $r=$ $\lceil(t-1) /(q-2)\rceil-1$ in Theorem 3.5. Therefore to prove the statement, by Theorem 3.5 it suffices to prove that $\left|\mathcal{K}_{r}(t)\right| \leq q^{\left(1-\delta_{2}\right) n}$. We have

$$
\begin{aligned}
\left|\mathcal{K}_{r}(t)\right| & =\left(\sum_{i=0}^{r}(q-1)^{i}\left(\begin{array}{c}
t+2 r \\
i
\end{array}\right)\right) q^{n-t-2 r}=S_{q}\left(\frac{r}{t+2 r}, t+2 r\right) q^{n-t-2 r} \\
& \leq q^{(1-1 / 125)(t+2 r)} q^{n-t-2 r}=q^{n-(t+2 r) / 125} \leq q^{(1-\epsilon / 125) n},
\end{aligned}
$$

using Proposition 3.3 in the first inequality and that $\epsilon n \leq t<t+2 r$ in the second. Taking $\delta_{2}(\epsilon)=\epsilon / 125$ completes the proof.

The next lemma is an analogue of Lemma 2.2 for subsets of $[q]^{n}$ and can be proved similarly.

Lemma 3.7. For $i=1,2$, suppose that $n_{i}, d_{i} \in \mathbb{N}$ and $p_{i} \in(0,1)$ are such that if $\mathcal{C}_{i} \subset[q]^{n_{i}}$ with $d_{i} \notin d\left(\mathcal{C}_{i}\right)$, then $\left|\mathcal{C}_{i}\right| \leq p_{i} q^{n_{i}}$. Suppose that $t \in \mathbb{N}$ satisfies $q^{-2 n_{1}}>p_{2}^{t}$. Then any set $\mathcal{C} \subset[q]^{n_{1}+n_{2}}$ with $d_{1}+d_{2} \notin d(\mathcal{C})$ satisfies $|\mathcal{C}| \leq\left(2 p_{1}\right)^{1 / t} q^{n_{1}+n_{2}}$. 
We are now ready for the proof of Theorem 1.3 ,

Proof of Theorem 1.3 . Let $\mathcal{C} \subset[q]^{n}$ with $|\mathcal{C}|=\alpha q^{n}$ where $q \geq 3$ and suppose that for some $d \in[\epsilon n,(1-\epsilon) n]$ we have $d \notin d(\mathcal{C})$. We wish to show that $\alpha \leq q^{-\delta n}$ where $\delta=\delta(\epsilon)>0$. By taking $\delta$ sufficiently small, we can assume that the result holds for $n<n_{0}(\epsilon)$, so we will assume that $n \geq n_{0}$. The proof will split into two cases, according as $d \in\left[\epsilon n, \frac{11}{20} n\right]$ or $d \in\left[\frac{11}{20} n,(1-\epsilon) n\right]$.

Case 1: $d \in\left[\epsilon n, \frac{11}{20} n\right]$. We will suppose that $d$ is odd, as the case of even $d$ is similar. As $d \geq \epsilon n$, provided $n \geq n_{0}(\epsilon)$, Theorem 2.5 allows us to write $d=d_{1}+d_{2}+d_{3}$ where $d_{i}$ are primes with $\left|d_{i}-d / 3\right| \leq \epsilon n / 100$. Also, partition $n$ as a sum of naturals $n=n_{1}+n_{2}+n_{3}$ where $\left|n_{i}-n / 3\right| \leq 1$ with $n_{1} \geq n_{2} \geq n_{3}$. For $n \geq n_{0}(\epsilon)$ this gives that for all $i \in[3]$ we have

$$
d_{i} \geq d / 3-\epsilon n / 100 \geq \epsilon n / 3-\epsilon n / 100 \geq \epsilon n_{i} / 2
$$

and

$$
d_{i} \leq d / 3+\epsilon n / 100 \leq 11 n / 60+\epsilon n / 100<3 n_{i} / 5 .
$$

Now set $V_{1}=\left[n_{1}\right], V_{2}=\left[n_{1}+1, n_{1}+n_{2}\right]$ and $V_{3}=\left[n_{1}+n_{2}+1, n\right]$. For each $i=1,2,3$ we have that $d_{i}$ is prime and by (3.2) and (3.3) it satisfies $\epsilon\left|V_{i}\right| / 2 \leq d_{i} \leq 3\left|V_{i}\right| / 5$. This gives that the hypotheses of Corollary 3.4 are satisfied (taking $\epsilon / 2$ in place of $\epsilon)$, and therefore any code $\mathcal{C}_{i} \subset[q]^{V_{i}}$ with $d_{i} \notin d\left(\mathcal{C}_{i}\right)$ satisfies $\left|\mathcal{C}_{i}\right| \leq p_{i} q^{n_{i}}$ where $p_{i}=q^{-\delta_{1}(\epsilon / 2) n_{i}}$.

We now use these bounds to obtain an upper bound on the size of codes $\mathcal{B} \subset$ $[q]^{V_{1} \cup V_{2}}$ with $d_{+}:=d_{1}+d_{2} \notin d_{H}(\mathcal{B})$ using Lemma 3.7. We first claim that taking $t_{1}=\left\lceil 4 / \delta_{1}(\epsilon / 2)\right\rceil$, we have $q^{-2 n_{1}}>p_{2}^{t_{1}}$. Indeed,

$$
p_{2}^{t_{1}}=q^{-\delta_{1}(\epsilon / 2) n_{2} t_{1}} \leq q^{-\delta_{1}(\epsilon / 2) n_{2}\left(\frac{4}{\delta_{1}(\epsilon / 2)}\right)}=q^{-4 n_{2}}<q^{-2 n_{1}},
$$

since $n_{2}>n_{1} / 2$. Lemma 3.7 then shows that any code $\mathcal{B} \subset[q]^{V_{1} \cup V_{2}}$ with $d_{+} \notin d(\mathcal{B})$ satisfies $|\mathcal{B}| \leq \alpha_{1} q^{n_{1}+n_{2}}$ where $\alpha_{1}=\left(2 q^{-\delta_{1}(\epsilon / 2) n_{1}}\right)^{1 / t_{1}}$.

To complete the proof we now repeat the previous argument to obtain a bound for codes $\mathcal{C} \subset[q]^{V_{1} \cup V_{2} \cup V_{3}}=[q]^{n}$ with $d=d_{+}+d_{3} \notin d_{H}(\mathcal{C})$. We first claim that setting $t_{2}=\left\lceil 4 t_{1} / \delta_{1}(\epsilon / 2)\right\rceil$ we have $\alpha_{1}^{t_{2}}<q^{-2 n_{3}}$. Indeed,

$$
\alpha_{1}^{t_{2}}=\left(2 q^{-\delta_{1}(\epsilon / 2) n_{1}}\right)^{t_{2} / t_{1}} \leq\left(2 q^{-\delta_{1}(\epsilon / 2) n_{1}}\right)^{4 / \delta_{1}(\epsilon / 2)}=2^{4 / \delta_{1}(\epsilon / 2)} q^{-4 n_{1}}<q^{-2 n_{3}} .
$$

The last inequality holds since $2^{4 / \delta_{1}(\epsilon / 2)}<2^{2 n / 3} \leq q^{2 n / 3} \leq q^{2 n_{1}}$ for $n \geq n_{0}(\epsilon) \geq$ $2 / \delta_{1}(\epsilon / 2)$.

Now any code $\mathcal{B} \subset[q]^{V_{1} \cup V_{2}}$ with $d_{+} \notin d_{H}(\mathcal{B})$ satisfies $|\mathcal{B}| \leq \alpha_{1} q^{n_{1}+n_{2}}$, and any code $\mathcal{C}_{3} \subset[q]^{V_{3}}$ with $d_{3} \notin d\left(\mathcal{C}_{3}\right)$ satisfies $\left|\mathcal{C}_{3}\right| \leq p_{3} q^{n_{3}}$ where $p_{3}=q^{-\delta_{1}(\epsilon / 2) n_{3}}$ and $\alpha_{1}^{t_{2}}<q^{-2 n_{3}}$. Therefore by Lemma 3.7 any code $\mathcal{C} \subset[q]^{V_{1} \cup V_{2} \cup V_{3}}=[q]^{n}$ with $d=d_{+}+d_{3} \notin d(\mathcal{C})$ satisfies $|\mathcal{C}| \leq \alpha_{2} q^{n}$ where

$$
\begin{aligned}
\alpha_{2} & =\left(2 p_{3}\right)^{1 / t_{2}} \leq\left(2 q^{-\delta_{1}(\epsilon / 2) n_{3}}\right)^{1 / t_{2}}=2^{1 / t_{2}} q^{-\delta_{1}(\epsilon / 2) n_{3} / t_{2}} \\
& \leq 2 q^{-\delta_{1}(\epsilon / 2) n / 4 t_{2}} \leq q^{-\delta_{1}(\epsilon / 2) n / 8 t_{2}}=q^{-\delta_{3}(\epsilon) n}
\end{aligned}
$$

where $\delta_{3}(\epsilon):=\delta_{1}(\epsilon / 2) / 8 t_{2}$. The last inequality here holds since for $n>n_{0}(\epsilon)$ we have $2 \leq q^{\delta_{1}(\epsilon / 2) n / 8 t_{2}}$.

Case 2: $d \in\left[\frac{11}{20} n,(1-\epsilon) n\right]$. We will prove this case using Case 1 above. The proof will work as follows. We will choose a partition $[n]=V_{1} \cup V_{2}$ and use dependent random choice to find a large subset $X \subset[q]^{V_{1}}$ such that for each pair of elements 
$x, x^{\prime} \in X$, the set of common extensions $y \in[q]^{V_{2}}$ with $x \circ y, x^{\prime} \circ y \in \mathcal{C}$ is also large. We can then apply Corollary 3.6 to find a pair $x, x^{\prime} \in X$ at Hamming distance $d^{\prime} \approx\left|V_{1}\right|$. Provided $\left|V_{1}\right|$ is carefully chosen, this will ensure that $d-d^{\prime} \leq 3\left|V_{2}\right| / 5$. We may then apply Case 1 to the set of extensions of $x$ and $x^{\prime}$ to find a pair $y, y^{\prime}$ with $d_{H}\left(y, y^{\prime}\right)=d-d^{\prime}$. Then $x \circ y, x^{\prime} \circ y^{\prime} \in \mathcal{C}$ lie at Hamming distance $d$.

Let $\delta_{3}$ denote the same function as in Case 1 and let $\delta_{2}$ denote the function in Corollary 3.6. Choose $n_{1} \in[n]$ such that

$$
\left|\frac{29}{40} n_{1}+\frac{11}{40} n-d\right| \leq 1
$$

As $d \geq \frac{11}{20} n$, this gives $n / 4 \leq n_{1} \leq(1-\epsilon) n$. Take $t=\left\lceil 2 / \epsilon \delta_{3}(1 / 4)\right\rceil$ and $\delta_{4}(\epsilon)=$ $\delta_{2}(\epsilon / 4) / 8 t>0$. We will show that if $\mathcal{C} \subset[q]^{n}$ where $|\mathcal{C}|=\alpha q^{n}$ with $\alpha>q^{-\delta_{4}(\epsilon) n}$, then $\mathcal{C}$ contains two words at Hamming distance $d$.

To begin, partition $[n]=V_{1} \cup V_{2}$ where $V_{1}=\left[n_{1}\right]$ and $V_{2}=\left[n_{1}+1, n\right]$. We set $n_{2}=n-n_{1}=\left|V_{2}\right|$. As in Lemma 2.2, view the elements of $\mathcal{C}$ as edges of a bipartite graph $G=(X, Y, E)$ with bipartition $X=[q]^{V_{1}}$ and $Y=[q]^{V_{2}}$, where $x y \in E(G)$ if $x \circ y \in \mathcal{C}$. Clearly $|E(G)|=\alpha|X||Y|$. Apply Lemma 2.1 to $G$ with $t$ as above to find a set $X^{\prime} \subset X$ with

$$
\begin{aligned}
\left|X^{\prime}\right|=\alpha^{t}|X| / 2 & >q^{-\delta_{4}(\epsilon) t n} q^{n_{1}} / 2=q^{-\delta_{2}(\epsilon / 4) n / 8} q^{n_{1}} / 2 \\
& \geq q^{-\delta_{2}(\epsilon / 4) n / 4} q^{n_{1}} \geq q^{\left(1-\delta_{2}(\epsilon / 4)\right) n_{1}}
\end{aligned}
$$

(using $q^{\delta_{2}(\epsilon / 4) n / 8} \geq 2$ for $n \geq n_{0}(\epsilon)$ and $n_{1} \geq n / 4$ ) such that all distinct $x, x^{\prime}$ in $X^{\prime}$ share at least $\alpha|X|^{-1 / t}|Y|$ common neighbours in $Y$. By Corollary 3.6 (with $\epsilon / 4$ in place of $\epsilon)$ there exists $x, x^{\prime} \in X^{\prime}$ with $d_{H}\left(x, x^{\prime}\right)=d^{\prime}$, with $d^{\prime}$ satisfying $(1-\epsilon / 4) n_{1} \leq d^{\prime} \leq n_{1}$. Let $\mathcal{B} \subset[q]^{V_{2}}$ denote the set of common extensions of $x, x^{\prime}$ in $Y$. We have

$$
\begin{aligned}
\log _{q}|\mathcal{B}| & \geq \log _{q}\left(\alpha|X|^{-1 / t}|Y|\right)>\log _{q}\left(q^{-\delta_{4}(\epsilon) n} q^{-n_{1} / t} q^{n_{2}}\right) \\
& =-\delta_{4}(\epsilon) n-n_{1} / t+n_{2}>-2 n_{1} / t+n_{2} \\
& \geq-2 n_{1} \epsilon \delta_{3}(1 / 4) / 2+n_{2}=-\epsilon \delta_{3}(1 / 4) n_{1}+n_{2} \\
& =-\delta_{3}(1 / 4) n_{2}\left(\frac{\epsilon n_{1}}{n_{2}}\right)+n_{2} \geq\left(1-\delta_{3}(1 / 4)\right) n_{2} .
\end{aligned}
$$

The final inequality used $n_{1} / n_{2}=n_{1} /\left(n-n_{1}\right) \leq 1 / \epsilon$ since $n_{1} \leq(1-\epsilon) n$. Therefore $|\mathcal{B}|>q^{\left(1-\delta_{3}(1 / 4)\right) n_{2}}$. As $d^{\prime} \in\left[(1-\epsilon / 4) n_{1}, n_{1}\right]$ and from (3.4) we have $\mid\left(d-n_{1}\right)-$ $\frac{11}{40} n_{2}|=| \frac{29}{40} n_{1}+\frac{11}{40} n-d \mid \leq 1$, we find that

$$
\begin{aligned}
d-d^{\prime} & \in\left[d-n_{1}, d-(1-\epsilon / 4) n_{1}\right] \\
& =\left[d-n_{1},\left(d-n_{1}\right)+\epsilon n_{1}\right] \\
& \subset\left[\frac{11}{40} n_{2}-1, \frac{11}{40} n_{2}+\epsilon n_{1} / 4\right] \\
& \subset\left[\frac{1}{4} n_{2}, \frac{11}{20} n_{2}\right] .
\end{aligned}
$$

Here we again used $n_{1} / n_{2}=n_{1} /\left(n-n_{1}\right) \leq 1 / \epsilon$. This shows that $\mathcal{B} \subset[q]^{V_{2}}$ satisfies $|\mathcal{B}|>q^{\left(1-\delta_{3}(1 / 4)\right) n_{2}}$. By definition of $\delta_{3}$, there exists a pair $y, y^{\prime} \in \mathcal{B}$ with $d_{H}\left(y, y^{\prime}\right)=d-d^{\prime}$. But this gives $x \circ y, x^{\prime} \circ y^{\prime} \in \mathcal{C}$. As $d_{H}\left(x \circ y, x^{\prime} \circ y^{\prime}\right)=d$ this completes the proof of this case.

Taking $\delta(\epsilon)=\min \left(\delta_{3}(\epsilon), \delta_{4}(\epsilon)\right)$ completes the proof of the theorem. 


\section{WEAK SUNFLOWERS IN $[q]^{n}$}

In this section, we will prove Theorem 1.6. For convenience, we will assume that $n$ is a multiple of $k$ with $n=k m$; this assumption can easily be removed. Set $V_{i}=[(i-1) m+1, i m]$ for all $i \in[k]$. We will prove by induction on $k$ that given $\epsilon>0$ and $d \in[\epsilon m,(1-\epsilon) m]$ (with $d$ even if $q=2$ ), there exists $\delta^{\prime}=\delta^{\prime}(\epsilon, k)>0$ with the following property: for any set $\mathcal{C} \subset[q]^{n}$ with $|\mathcal{C}|>q^{\left(1-\delta^{\prime}\right) n}$, there exists $x_{i}, y_{i} \in[q]^{V_{i}}$ for $i \in[k]$ with $d_{H}\left(x_{i}, y_{i}\right)=d$, such that $z_{1} \circ \cdots \circ z_{k} \in \mathcal{C}$ for any choice of $z_{i} \in\left\{x_{i}, y_{i}\right\}$. This will complete the proof as taking

$$
v_{i}=x_{1} \circ \cdots \circ x_{i-1} \circ y_{i} \circ x_{i+1} \circ \cdots \circ x_{k},
$$

the set $\left\{v_{1}, \ldots, v_{k}\right\}$ is a weak sunflower with $k$ petals contained in $\mathcal{C}$.

The case when $k=1$ follows immediately from Theorem 1.3 , so we will assume by induction that the result holds for $k-1$ and prove it for $k$. Let $W_{1}=\bigcup_{i=2}^{k} V_{i}$ so that $[n]=V_{1} \cup W_{1}$. Letting $t=\left\lceil 2 /\left((k-1) \delta^{\prime}(\epsilon, k-1)\right)\right\rceil$, we claim that we can take $\delta^{\prime}=\delta^{\prime}(\epsilon, k)=\delta(\epsilon) / 2 k t$, where $\delta(\epsilon)$ is as in Theorem 1.3. Indeed, as in the proof of Lemma 2.2 view elements of $[q]^{n}$ as edges of a bipartite graph $G=\left([q]^{V_{1}},[q]^{W_{1}}, E\right)$ in which $x y \in E(G)$ if $x \circ y \in \mathcal{C}$. Then if $|\mathcal{C}|=|E(G)|=\alpha q^{n}$ where $\alpha>q^{-\delta^{\prime} n}$, by Lemma 2.1, there exists a set $\mathcal{C}_{1} \subset[q]^{V_{1}}$ with

$$
\left|\mathcal{C}_{1}\right| \geq \alpha^{t} q^{m} / 2 \geq q^{-\delta(\epsilon) n / 2 k} q^{m} / 2=q^{(1-\delta(\epsilon) / 2) m} / 2 \geq q^{(1-\delta(\epsilon)) m}
$$

with any two elements in $\mathcal{C}_{1}$ sharing at least

$$
\alpha q^{-m / t} q^{(k-1) m}>q^{-\frac{\delta(\epsilon) n}{2 k t}-\frac{m}{t}} q^{(k-1) m} \geq q^{-\frac{2 m}{t}} q^{(k-1) m} \geq q^{(1-\delta(\epsilon, k-1))(k-1) m}
$$

common neighbours in $G$. But then, by Theorem 1.3, $\mathcal{C}_{1}$ must contain elements $x_{1}$ and $x_{2}$ with $d_{H}\left(x_{1}, x_{2}\right)=d$. Also, by the induction hypothesis for $k-1$, we find $x_{i}, y_{i} \in[q]^{V_{i}}$ for all $i \in[2, k]$ with $d_{H}\left(x_{i}, y_{i}\right)=d$ such that all elements of the set

$$
\left\{z_{2} \circ \cdots \circ z_{k}: z_{i} \in\left\{x_{i}, y_{i}\right\} \text { for all } i \in[2, k]\right\}
$$

are common neighbours of both $x_{1}$ and $y_{1}$. But by definition of $G$, this means that $z_{1} \circ \cdots \circ z_{l} \in \mathcal{C}$ for any choice of $z_{i} \in\left\{x_{i}, y_{i}\right\}$ for all $i \in[k]$, as claimed.

\section{Forbidding DistanCES BETWEen PAIRS OF SETS IN $[q]^{n}$}

Proof of Theorem 1.8. Given $\epsilon$ we will take $\delta^{\prime}(\epsilon)=\delta(\epsilon / 2) / 2$, where $\delta(\epsilon / 2)$ is as in Theorem 1.3 and $\gamma=\min \left(\epsilon / 2, \frac{\delta(\epsilon / 2)}{16 \log (1 / \delta(\epsilon / 2))}\right)$. Let $q \geq 3$ and suppose that $\mathcal{C}, \mathcal{D} \subset[q]^{n}$ with $|\mathcal{C}| \geq q^{\left(1-\delta^{\prime}\right) n}$ and such that for all $x \in \mathcal{C}$ there is $y \in \mathcal{D}$ with $d_{H}(x, y) \leq \gamma n$. Suppose $d \in(\epsilon n,(1-\epsilon) n)$. We will show that there exist $x \in \mathcal{C}$ and $y \in \mathcal{D}$ with $d_{H}(x, y)=d$.

From the statement, for all $x \in \mathcal{C}$ there is some $y_{x} \in \mathcal{D}$ with $d_{H}\left(x, y_{x}\right) \leq \gamma n$. By pigeonholing, there must be a set $T \subset\left(\begin{array}{c}{[n]} \\ \gamma n\end{array}\right)$ and a subset $\mathcal{C}^{\prime} \subset \mathcal{C}$ with $\left|\mathcal{C}^{\prime}\right| \geq$ $|\mathcal{C}| /\left(\begin{array}{c}n \\ \gamma n\end{array}\right) \geq|\mathcal{C}| 2^{-H(\gamma) n}$ with the property that, for all $x \in \mathcal{C}^{\prime}$, we have $\{i \in[n]$ : $\left.(x)_{i} \neq\left(y_{x}\right)_{i}\right\} \subset T$. There are at most $q^{\gamma n}$ choices for both $\left.x\right|_{T}$ and $\left.y_{x}\right|_{T}$, so again by pigeonholing we find $\mathcal{C}^{\prime \prime} \subset \mathcal{C}^{\prime}$ with $\left|\mathcal{C}^{\prime \prime}\right| \geq\left|\mathcal{C}^{\prime}\right| / q^{2 \gamma n}$ and vectors $f_{0}, g_{0} \in[q]^{T}$ such that $\left.x\right|_{T}=f_{0}$ and $\left.y_{x}\right|_{T}=g_{0}$ for all $x \in \mathcal{C}^{\prime \prime}$. Let $d_{H}\left(f_{0}, g_{0}\right)=t \leq \gamma n \leq \epsilon n / 2$. Now by choice of $\gamma$, we have $H(\gamma) \leq \delta(\epsilon / 2) / 4$ and $\gamma<\delta(\epsilon / 2) / 8$ and so

$$
\left|\mathcal{C}^{\prime \prime}\right| \geq|\mathcal{C}| 2^{-H(\gamma) n} q^{-2 \gamma n} \geq q^{(1-\delta(\epsilon / 2)) n} .
$$


Therefore, since $\epsilon n / 2 \leq d-\gamma n \leq d-t \leq(1-\epsilon) n$ by Theorem 1.3 there are $x, x^{\prime} \in \mathcal{C}^{\prime \prime}$ with $d_{H}\left(x, x^{\prime}\right)=d-t$. But then

$$
d_{H}\left(x^{\prime}, y_{x}\right)=\underbrace{d_{H}\left(x, y_{x}\right)}_{\text {distance in } T}+\underbrace{d_{H}\left(x^{\prime}, x\right)}_{\text {distance in }[n] \backslash T}=d_{H}\left(f_{0}, g_{0}\right)+d_{H}\left(x^{\prime}, x\right)=d \text {. }
$$

As $x^{\prime} \in \mathcal{C}$ and $y_{x} \in \mathcal{D}$, this completes the proof.

We will now show that the conditions of Theorem 1.8 can be applied to $\mathcal{C}, \mathcal{D} \subset$ $[q]^{n}$ with $|\mathcal{C}|,|\mathcal{D}| \geq(q-\delta)^{n}$ provided $\delta=\delta(\epsilon, q)>0$ is sufficiently small, proving Corollary [1.9. Let $K_{q}^{n}$ denote the graph on vertex set $[q]^{n}$, in which $x, y \in[q]^{n}$ are adjacent if they differ on a single coordinate. Given a set $\mathcal{C} \subset[q]^{n}$, let $N^{(t)}(\mathcal{C})=\left\{x \in[q]^{n}: d_{H}\left(x, x^{\prime}\right) \leq t\right.$ for some $\left.x^{\prime} \in \mathcal{C}\right\}$. The following result gives an approximate vertex isoperimetric theorem for $K_{q}^{n}$ (see [18] or [3]).

Theorem 5.1. For all $q \geq 2$ and $\gamma>0$, there exists $\delta^{\prime \prime}=\delta^{\prime \prime}(\gamma, q)>0$ such that the following holds. Any set $\mathcal{C} \subset[q]^{n}$ with $|\mathcal{C}| \geq\left(q-\delta^{\prime \prime}\right)^{n}$ satisfies $\left|N^{(\gamma n)}(\mathcal{C})\right|>$ $q^{n}-\left(q-\delta^{\prime \prime}\right)^{n}$.

Proof of Corollary 1.9. Let $q \geq 3$ and $\epsilon>0$. We wish to show that, given any $\mathcal{C}, \mathcal{D} \subset[q]^{n}$ with $|\mathcal{C}||\mathcal{D}|>(q-\delta)^{2 n}$ where $\delta=\delta(\epsilon, q)>0$ is sufficiently small, for any $d \in(\epsilon n,(1-\epsilon) n)$ there exist $x \in \mathcal{C}$ and $y \in \mathcal{D}$ with $d_{H}(x, y)=d$. By taking $\delta$ to be sufficiently small it suffices to prove the result for $n \geq n_{0}(\epsilon, q)$. Given our value of $\epsilon$, let $\delta^{\prime}>0$ and $\gamma>0$ be as in Theorem 1.8. We will take $\delta=\delta^{\prime \prime}(\gamma, q) / 4$, with $\delta^{\prime \prime}(\gamma, q)$ as in Theorem 5.1 .

First note that as $|\mathcal{C}|,|\mathcal{D}| \leq q^{n}$ we have $|\mathcal{C}|,|\mathcal{D}| \geq \frac{(q-\delta)^{2 n}}{q^{n}}>(q-2 \delta)^{n}$. Take $\mathcal{C}^{\prime} \subset \mathcal{C}$ to be the set $\mathcal{C}^{\prime}=\left\{x \in \mathcal{C}: \exists y \in \mathcal{D}\right.$ with $\left.d_{H}(x, y) \leq \gamma n\right\}$. By Theorem 1.8, it suffices to show that $\left|\mathcal{C}^{\prime}\right|>q^{\left(1-\delta^{\prime}\right) n}$. Suppose for contradiction that this is not the case. Then for $n \geq n_{0}$, we have

$$
\left|\mathcal{C} \backslash \mathcal{C}^{\prime}\right| \geq|\mathcal{C}|-\left|\mathcal{C}^{\prime}\right|>(q-2 \delta)^{n}-q^{\left(1-\delta^{\prime}\right) n} \geq(q-4 \delta)^{n}=\left(q-\delta^{\prime \prime}\right)^{n} .
$$

Now by Theorem 5.1 we have $\left|N^{(\gamma n)}\left(\mathcal{C} \backslash \mathcal{C}^{\prime}\right)\right|>q^{n}-\left(q-\delta^{\prime \prime}\right)^{n}$. However, by definition of $\mathcal{C}^{\prime}$ we have $\mathcal{D} \cap N^{(\gamma n)}\left(\mathcal{C} \backslash \mathcal{C}^{\prime}\right)=\emptyset$. As $|\mathcal{D}| \geq(q-2 \delta)^{n}=\left(q-\delta^{\prime \prime} / 2\right)^{n}>\left(q-\delta^{\prime \prime}\right)^{n}$ this is a contradiction, and it completes the proof of the corollary.

\section{Supersaturated version of Theorem 1.3}

In this section we prove Theorem 1.10 which gives a supersaturated version of Theorem 1.3. A rough outline of the proof is as follows. We will assume for contradiction that we are given a large set $\mathcal{C}$, as in Theorem 1.10, which contains a few pairs at Hamming distance $d$, for some $\epsilon n \leq d \leq(1-\epsilon) n$. By fixing some coordinate entries from $[n]$ and restricting others, we will find a large subset $\mathcal{E}^{\prime} \subset$ $[r]^{V_{1}}$ with $r \leq q$ and $\left(1+\alpha^{\prime}\right) d \leq\left|V_{1}\right| \leq n$ which contains no pairs at distance $d$. However, if $\mathcal{E}^{\prime}$ is a sufficiently large subset $[r]^{V_{1}}$, this will contradict Theorem 1.3 .

A technical complication which occurs in our proof is that on reducing from $[q]^{n}$ to $[r]^{V_{1}}$, it is possible that $q \geq 3$ and $d$ is odd, but that after restricting we end up with $r=2$. Note that in this case, we cannot apply Theorem 1.3 to $\mathcal{E}^{\prime}$, as only even distances can be guaranteed in $[2]^{V_{1}}$. To get around this obstacle, we first prove the case when $d$ is even separately and then deduce the odd case from it by an application of dependent random choice. 
Proof of Theorem 1.10, Set $\alpha=\eta /(16 \log (16 / \eta))$ and $\delta^{\prime}=\eta \epsilon \delta(\alpha / 2) / 8$, where $\delta$ is as in Theorem 1.3. Also set $m=\alpha n$ and $r=\max \left\{\left\lfloor q^{\eta / 4}\right\rfloor, 2\right\}$. Let $\mathcal{C} \subset[q]^{n}$ with $|\mathcal{C}|>q^{\left(1-\delta^{\prime}\right) n}$. We will show that given $d$ with $\epsilon n \leq d \leq(1-\epsilon) n$, the code $\mathcal{C}$ contains at least $\left(\begin{array}{l}n \\ d\end{array}\right)(q-1)^{d}|\mathcal{C}| q^{-\eta n}$ pairs $x, y \in \mathcal{C}$ with $d_{H}(x, y)=d$. As discussed above, we start by giving the proof in the case where $d$ is even.

Let $N$ denote the number of pairs $\{x, y\}$ with $x, y \in \mathcal{C}$ such that $d_{H}(x, y)=d$. Make the following selection of random choices:

- choose a partition of $[n]=V_{1} \cup V_{2}$ with $\left|V_{1}\right|=d+m$ and $\left|V_{2}\right|=n-d-m$ uniformly at random;

- for each $i \in V_{1}$, choose a subset $Q_{i} \subset[q]$ of size $r$ uniformly at random;

- for each $i \in V_{2}$, choose an element $q_{i} \in[q]$ uniformly at random.

We will say that an element $x \in \mathcal{C}$ is a captured element if $x_{i} \in Q_{i}$ for all $i \in V_{1}$ and $x_{j}=q_{j}$ for all $j \in V_{2}$. Let $\mathcal{E} \subset \mathcal{C}$ denote the set of captured elements. We also say that a pair $\{x, y\} \in \mathcal{C}^{(2)}$ is a captured d-pair if $x, y \in \mathcal{E}, d_{H}(x, y)=d$ and $V_{2} \subset \operatorname{Agree}(x, y)$.

Let $X$ and $Y$ denote the random variables which count the number of captured elements and the number of captured $d$-pairs respectively. Clearly, given $x \in \mathcal{C}$, we have $\mathbb{P}(x \in \mathcal{E})=r^{d+m} / q^{n}$. Therefore we have

$$
\mathbb{E}(X)=\frac{r^{d+m}|\mathcal{C}|}{q^{n}} .
$$

For a fixed pair $x, y \in \mathcal{C}$ with $d_{H}(x, y)=d$ we have

$$
\mathbb{P}(x, y \text { form a captured } d \text {-pair })=\frac{\left(\begin{array}{c}
d+m \\
d
\end{array}\right)}{\left(\begin{array}{c}
n \\
d
\end{array}\right)}\left(\frac{\left(\begin{array}{l}
r \\
2
\end{array}\right)}{\left(\begin{array}{l}
q \\
2
\end{array}\right)}\right)^{d}\left(\frac{r}{q}\right)^{m} q^{-(n-d-m)} .
$$

To see this we justify one term at a time. The first term is the probability that the $d$ coordinates on which $x$ and $y$ differ are included in $V_{1}$. The second term is the probability that the entries $x_{i}$ and $y_{i}$ are included in $Q_{i}$ where they differ. The third term is the probability that the remaining (common) entries of $x_{i}=y_{i}$ in $V_{1}$ are included in $Q_{i}$ and the last term is the probability that $q_{i}=x_{i}$ for $i \in V_{2}$.

Suppose for contradiction that $N<\left(\begin{array}{l}n \\ d\end{array}\right)(q-1)^{d}|\mathcal{C}| q^{-\eta n}$. Then

$$
\begin{aligned}
\mathbb{E}(Y) & =\left(\frac{r}{q}\right)^{m}\left(\frac{r(r-1)}{q(q-1)}\right)^{d} q^{-(n-d-m)} \frac{\left(\begin{array}{c}
d+m \\
m
\end{array}\right)}{\left(\begin{array}{c}
n \\
d
\end{array}\right)} N \\
& <\left(\begin{array}{c}
d+m \\
m
\end{array}\right) \frac{r^{d+m}(r-1)^{d}}{q^{n}}|\mathcal{C}| q^{-\eta n} \\
& \leq\left(\frac{\left(\begin{array}{c}
n \\
m
\end{array}\right)(r-1)^{n}}{q^{\eta n}}\right) \frac{r^{d+m}|\mathcal{C}|}{q^{n}} .
\end{aligned}
$$

Now for $\alpha \leq \eta /(16 \log (16 / \eta))$ we have $H(\alpha) \leq \eta / 4$ and so $\left(\begin{array}{c}n \\ m\end{array}\right) \leq 2^{H(\alpha) n}<q^{\eta n / 4}$. Since we also have $(r-1) \leq \max \left\{q^{\eta / 4}, 1\right\}=q^{\eta / 4}$ we have

$$
\mathbb{E}(Y) \leq \frac{1}{q^{\eta n / 2}} \frac{r^{d+m}|\mathcal{C}|}{q^{n}} \leq \frac{r^{d+m}|\mathcal{C}|}{2 q^{n}}
$$

for $n \geq n_{0} \geq 2 / \eta$. Combined with (6.1), as $|\mathcal{C}| \geq q^{\left(1-\delta^{\prime}\right) n}$, this gives

$$
\mathbb{E}(X-Y) \geq \frac{r^{d+m}|\mathcal{C}|}{2 q^{n}} \geq \frac{r^{d+m} q^{-\delta^{\prime} n}}{2} .
$$


But $q^{-\delta^{\prime} n}=\left(q^{\eta / 4}\right)^{-\delta(\alpha / 2) \epsilon n / 2} \geq r^{-\delta(\alpha / 2) \epsilon n / 2} \geq r^{-\delta(\alpha / 2)(d+m) / 2}$ as $d \geq \epsilon n$. This shows that

$$
\mathbb{E}(X-Y) \geq \frac{r^{(1-\delta(\alpha / 2) / 2)(d+m)}}{2}>r^{(1-\delta(\alpha / 2))(d+m)} .
$$

The second inequality here holds since $r^{\delta(\alpha / 2)(d+m) / 2} \geq r^{\delta(\alpha / 2) \epsilon n / 2} \geq 2$ for $n \geq n_{0}$. Fix choices of $V_{1}, V_{2}, Q_{i}$ for all $i \in V_{1}$ and $q_{i}$ for $i \in V_{2}$ such that $X-Y$ is at least this big. Now remove one element from every captured $d$-pair in $\mathcal{E}$. By (6.3), this leaves a set $\mathcal{E}^{\prime} \subset \mathcal{E}$ with

$$
\left|\mathcal{E}^{\prime}\right|>r^{(1-\delta(\alpha / 2))(d+m)}
$$

which contains no captured $d$-pairs.

Now $\mathcal{E}^{\prime}$ is a subset of $\prod_{i \in V_{1}} Q_{i} \times \prod_{j \in V_{2}}\left\{q_{j}\right\}$, and this product set is naturally identified with $[r]^{d+m}$. We also have

$$
\frac{\alpha}{2}(d+m) \leq d \leq\left(1-\frac{\alpha}{2}\right)(d+m) .
$$

Indeed, $\alpha(d+m) / 2 \leq d$ since $m \leq d$ and $\alpha \leq 1$ and $d \leq(1-\alpha / 2)(d+m)$ since $\alpha d / 2 \leq \alpha n / 2=m / 2 \leq(1-\alpha / 2) m$. But now since $\mathcal{E}^{\prime}$ does not contain a pair $(x, y)$ with $d_{H}(x, y)=d$, by Theorem 1.3 we have $\left|\mathcal{E}^{\prime}\right| \leq q^{(1-\delta(\alpha / 2))(d+m)}$. However this contradicts (6.4). Therefore we must have $N \geq\left(\begin{array}{l}n \\ d\end{array}\right)(q-1)^{d}|\mathcal{C}| q^{-\eta n}$, as required. This completes the proof of the case of $d$ even.

The case of $d$ odd and $q \geq 3$ can be deduced from the even case by dependent random choice. As it is similar to previous steps, we only outline the argument. Given $\epsilon$ and $\eta$ as in the statement we will assume that $\gamma$ and $\zeta$ are chosen sufficiently small so that various estimates against functions of $\epsilon$ and $\eta$ hold. First choose a partition of $[n]=V_{1} \cup V_{2}$, where $\left|V_{1}\right|=\gamma n$. By dependent random choice (see Lemma 2.1), provided that $|\mathcal{C}| \geq q^{(1-\delta) n}$ with $\delta=\delta(\gamma, \zeta)>0$ sufficiently small, there is a subset $\mathcal{S} \subset[q]^{V_{1}}$ with $|\mathcal{S}| \geq q^{(1-\zeta)\left|V_{1}\right|}$ such that for every pair $x, y \in \mathcal{S}$, the set

$$
\mathcal{C}_{x, y}:=\left\{z \in[q]^{V_{2}}: \text { with } x \circ z, y \circ z \in \mathcal{C}\right\}
$$

satisfies $\left|\mathcal{C}_{x, y}\right| \geq q^{(1-\zeta)\left|V_{2}\right|}$. Now as $\zeta$ is small, we can apply Theorem 1.3 to $\mathcal{S}$ to find $x, y \in \mathcal{S}$ with $d_{H}(x, y)=d^{\prime}$ odd - this is possible since $q \geq 3$. Taking $d^{\prime \prime}=d-d^{\prime}$, as $d^{\prime} \leq\left|V_{1}\right|=\gamma n$, we have

$$
\epsilon\left|V_{2}\right| / 2<\epsilon n-\gamma n \leq d^{\prime \prime} \leq(1-\epsilon) n \leq(1-\epsilon / 2)\left|V_{2}\right|,
$$

provided $\gamma$ was chosen with $\gamma<\epsilon / 4$.

We now use the even case proven above to guarantee that $\mathcal{C}_{x, y}$ contains many pairs at Hamming distance $d^{\prime \prime}$. Indeed, as $\left|\mathcal{C}_{x, y}\right| \geq q^{(1-\zeta)\left|V_{2}\right|}$ and $d^{\prime \prime}$ is even, if $\zeta$ was chosen so that $\zeta<\delta^{\prime}(\epsilon / 2, \eta / 2)$, the even case shows that $\mathcal{C}_{x, y}$ contains at least $\left(\begin{array}{c}\left|V_{2}\right| \\ d^{\prime \prime}\end{array}\right)(q-1)^{d^{\prime \prime}}\left|\mathcal{C}_{x, y}\right| q^{-\eta\left|V_{2}\right| / 2}$ pairs at Hamming distance $d^{\prime \prime}$. Expanding we find

$$
\begin{aligned}
\left(\begin{array}{c}
\left|V_{2}\right| \\
d^{\prime \prime}
\end{array}\right)(q-1)^{d^{\prime \prime}}\left|\mathcal{C}_{x, y}\right| q^{-\eta\left|V_{2}\right| / 2} \geq & \left(\begin{array}{l}
n \\
d
\end{array}\right) \frac{(d)_{d^{\prime}}}{(n)_{d^{\prime}}} \times(q-1)^{d} q^{-d^{\prime}} \\
& \times|\mathcal{C}| q^{-\zeta n-\gamma n} \times q^{-\eta n / 2} \\
\geq & \left(\begin{array}{l}
n \\
d
\end{array}\right)(q-1)^{d}|\mathcal{C}|\left(\frac{\epsilon}{2}\right)^{\gamma n} q^{-(\zeta+2 \gamma+\eta / 2) n} .
\end{aligned}
$$


The second inequality here holds since $\frac{(d)_{d^{\prime}}}{(n)_{d^{\prime}}} q^{-d^{\prime}} \geq(\epsilon / 2)^{\gamma n} q^{-\gamma n}$ holds for $d^{\prime} \leq \gamma n<$ $\epsilon n / 2$. For $\gamma$ and $\zeta$ sufficiently small in comparison with $\epsilon$ and $\eta$, this gives at least $\left(\begin{array}{l}n \\ d\end{array}\right)(q-1)^{d}|\mathcal{C}| q^{-\eta n}$ such pairs $z, z^{\prime} \in \mathcal{C}_{x, y}$. But for each of these pairs, we have $x \circ z, y \circ z^{\prime} \in \mathcal{C}$ and $d_{H}\left(x \circ z, y \circ z^{\prime}\right)=d^{\prime}+d^{\prime \prime}=d$. This completes the proof.

\section{Concluding Remarks}

In this paper we gave improved bounds on the size of codes and families of permutations with a forbidden distance. These bounds demonstrate the power of dependent random choice in forbidden distance problems, and we expect that the method will have many more applications in extremal set theory.

It remains an intriguing open problem to obtain a better upper bound on the size of maximum $l$-avoiding families $\mathcal{A} \subset \mathcal{P}[n]$. A natural construction is to take all sets that are 'large' or 'small', where 'large' sets have size at least $(n+l) / 2$ and 'small' sets have size less than $l$. (If $n+l$ is odd we can also add all sets of size $(n+l-1) / 2$ containing 1). For fixed $l$ and large $n$, Frankl and Füredi [13] proved that this is the unique extremal family.

However, much less is known when $l$ is comparable with $n$. Under the stronger condition of being $(l+1)$-intersecting, Katona [17] showed that the family of all large sets gives the optimal construction. Mubayi and Rödl [19] conjectured that for the $l$ avoiding problem, with any $\epsilon n<l<(1 / 2-\epsilon) n$, the same family of all large sets and all small sets as before should be approximately optimal, say up to a multiplicative factor of $2^{o(n)}$. They proved this when the $l$-avoiding condition is replaced with the stronger condition of having a small forbidden interval of intersections around $l$.

\section{ACKNOWLEDGEMENTS}

The authors would like to thank both referees for their careful reading of the paper and for several helpful comments which improved the presentation.

\section{REFERENCES}

[1] Rudolf Ahlswede and Levon H. Khachatrian, The diametric theorem in Hamming spaces-optimal anticodes, Adv. in Appl. Math. 20 (1998), no. 4, 429-449, DOI 10.1006/aama.1998.0588. MR1612850

[2] Noga Alon, Amir Shpilka, and Christopher Umans, On sunflowers and matrix multiplication, Comput. Complexity 22 (2013), no. 2, 219-243, DOI 10.1007/s00037-013-0060-1. MR3055780

[3] Béla Bollobás and Imre Leader, Compressions and isoperimetric inequalities, J. Combin. Theory Ser. A 56 (1991), no. 1, 47-62, DOI 10.1016/0097-3165(91)90021-8. MR.1082842

[4] László Babai, Hunter Snevily, and Richard M. Wilson, A new proof of several inequalities on codes and sets, J. Combin. Theory Ser. A 71 (1995), no. 1, 146-153, DOI 10.1016/00973165(95)90021-7. MR 1335782

[5] R. C. Baker and G. Harman, The three primes theorem with almost equal summands, R. Soc. Lond. Philos. Trans. Ser. A Math. Phys. Eng. Sci. 356 (1998), no. 1738, 763-780, DOI 10.1098/rsta.1998.0184. MR.1620832

[6] Harry Buhrman, Richard Cleve, and Avi Wigderson, Quantum vs. classical communication and computation, STOC '98 (Dallas, TX), ACM, New York, 1999, pp. 63-68. MR1731563

[7] Herman Chernoff, A measure of asymptotic efficiency for tests of a hypothesis based on the sum of observations, Ann. Math. Statistics 23 (1952), 493-507. MR0057518

[8] Péter Frankl and Mikhail Deza, On the maximum number of permutations with given maximal or minimal distance, J. Combinatorial Theory Ser. A 22 (1977), no. 3, 352-360. MR.0439648 
[9] P. Erdős, Problems and results in graph theory and combinatorial analysis, Proceedings of the Fifth British Combinatorial Conference (Univ. Aberdeen, Aberdeen, 1975), Congressus Numerantium, No. XV, Utilitas Math., Winnipeg, Man., 1976, pp. 169-192. MR0409246

[10] David Ellis, Forbidding just one intersection, for permutations, J. Combin. Theory Ser. A 126 (2014), 136-165, DOI 10.1016/j.jcta.2014.04.011. MR3213310

[11] Jacob Fox and Benny Sudakov, Dependent random choice, Random Structures Algorithms 38 (2011), no. 1-2, 68-99, DOI 10.1002/rsa.20344. MR.2768884

[12] P. Frankl, Orthogonal vectors in the $n$-dimensional cube and codes with missing distances, Combinatorica 6 (1986), no. 3, 279-285, DOI 10.1007/BF02579389. MR875296

[13] P. Frankl and Z. Füredi, On hypergraphs without two edges intersecting in a given number of vertices, J. Combin. Theory Ser. A 36 (1984), no. 2, 230-236, DOI 10.1016/00973165(84)90008-6. MR734980

[14] Peter Frankl and Vojtěch Rödl, Forbidden intersections, Trans. Amer. Math. Soc. 300 (1987), no. 1, 259-286, DOI 10.2307/2000598. MR871675

[15] P. Frankl and V. Rödl, A partition property of simplices in Euclidean space, J. Amer. Math. Soc. 3 (1990), no. 1, 1-7, DOI 10.1090/S0894-0347-1990-1020148-2. MR1020148

[16] P. Frankl and R. M. Wilson, Intersection theorems with geometric consequences, Combinatorica 1 (1981), no. 4, 357-368, DOI 10.1007/BF02579457. MR647986

[17] Gy. Katona, Intersection theorems for systems of finite sets, Acta Math. Acad. Sci. Hungar 15 (1964), 329-337. MR.0168468

[18] L. H. Harper, On an isoperimetric problem for Hamming graphs, Proceedings of the Conference on Optimal Discrete Structures and Algorithms-ODSA '97 (Rostock), Discrete Appl. Math. 95 (1999), no. 1-3, 285-309, DOI 10.1016/S0166-218X(99)00082-7. MR1708844

[19] Dhruv Mubayi and Vojtech Rödl, Specified intersections, Trans. Amer. Math. Soc. 366 (2014), no. 1, 491-504, DOI 10.1090/S0002-9947-2013-05877-1. MR3118403

[20] Jiří Sgall, Bounds on pairs of families with restricted intersections, Combinatorica 19 (1999), no. 4, 555-566, DOI 10.1007/s004939970007. MR:1773657

Mathematical Institute, University of Oxford, Oxford OX2 6GG, United Kingdom

E-mail address: Peter.Keevash@maths.ox.ac.uk

Mathematical Institute, University of Oxford, Oxford OX2 6GG, United Kingdom

E-mail address: Eoin.Long@maths.ox.ac.uk

Current address: School of Mathematical Sciences, Tel Aviv University, 69978 Tel Aviv, Israel

E-mail address: eoinlong@post.tau.ac.il 